\title{
A Network Measurement System for NFV Networks based on SDN
}

\author{
S.Sathishkumar ${ }^{1}$, S.Rajkumar ${ }^{2}$, V.Adarsh ${ }^{3}$, K.Hameed Musharaf ${ }^{4}$ \\ ${ }^{1}$ Assistant Professor, India, sathishkumar.it@adhiyamaan.in \\ ${ }^{2}$ Student, India, rajkumarsundar03@gmail.com \\ ${ }^{3}$ Student, India, benadarsh@gmail.com \\ ${ }^{4}$ Student, India, shafa.musraf@gmail.com
}

\begin{abstract}
As a promising innovation to totally change how we design, install and oversee different organizations, Software-based Network Function Virtualization (NFV) empowers hardware-free, adaptable, quick and proficient network service process. With the expanding prevalence of NFV, the Internet has likewise changed to be a hybrid environment where NFV-based network exists together with traditional gadgets. To ease our understanding, plan, assess and handling of such novel organization environment, there is an incredible requirement for new NFV-compatible measurement framework which is still missing till now. To overcome this issue, a framework, named Software Defined Network Measurement System (SDNMS) is introduced. First the architecture of SDNMS is proposed. In this design, a conventional strategy to portray the working method of the network measurement is defined. This technique can likewise be used to produce network measurement middle box (NMMB) in a particular location of the NFV network as per the customized description file, and to flexibly install the network measurement function. Besides, the innovation of virtual network measurement function (VNMF) joined with LXC is said to be shape NMMB function. Thirdly, a control strategy is introduced to control the beginning, stop, and update NMMB to produce a particular network measurement system. At last, a model of SDNMS with network observing function to screen network performance anomalies and locate fault is presented. Test results have shown that SDNMS design and related advancements are practical and successful to launch and control network measurement functions in NFV network. We trust SDNMS could give another strategy to experts to do network management and examine at the age of NFV.
\end{abstract}

Key words : Energy Reduction, Network Virtualization, SDN, SDN Controller.

\section{INTRODUCTION}

Software Defined Networks and Network Function Virtualization (SDN-NFV) have effectively raged the tele-contact market and impacted the Communication Service Providers (CSPs) to put resources into fabricate an adaptable, dexterous, and assertive network system. While it keeps on impacting CSP's techniques and running choices, CIO is battling to track down correct use cases utilizing SDN-NFV innovations being conveying business esteem. SDN-NFV begins as NULL normalization and sellers are creating their own items utilizing SDN. To beat the challenges in present network and to fabricate a combined system, a mixed configuration with actual and imaginary hardware is required. SDN-NFV solution provides E-E stack, empowering CSPs to convey service and network on-request to offer support inventories, online channels, charging, OSS/BSS stack, and harmony engine, while incorporating it with SDN controllers. Agile cloud solutions supported by APIs and request the executives, undertaking clients can utilize an omnichannel presentation layer to lay out and oversee flaws. Additionally, its helps CSPs in creating and monitoring the services capacities that convey constant charging and give the adaptability of offering a value plan dependent on utilization and membership

\section{LITERATURE SURVEY}

A. SDN and its Use-Cases - NV and NFV: A State-of-the-Art survey - Three ideas - (a) Program driven network by pure division of information and management of planes and (b) distributing organization framework gives busy network confinement, in enormous server center networks and (c) changing the capacities which generally run in the specific hardware, product acknowledge which suddenly spike in demand for ware workers - have acquired parcel of consideration by both Industry and examination local area over recent years. Those three ideas were extensively alluded as software defined networking (SDN), network virtualization (NV) and network functions virtualization (NFV). This paper gives a careful investigation of those three concepts, including SDN innovation which supplement organization virtualization and organization capacities virtualization. The review of SDN is trailed by examining when SDN assumes a critical part in NV and NFV. At long last, this work likewise attempts to investigate future headings in SDN dependents upon latest things. 
B.Software-Defined Network Virtualization --- An Architectural Framework for Integrating SDN and NFV for Service Provisioning in Future Networks - Software Defined Network (SDN) and Network Function Virtualization (NFV) are huge advancements in networking. Advancement by Network Function Virtualization and Software Defined Network shown solid collaboration between standards. Late exploration attempts have been made toward joining SDN and NFV to completely misuse the benefits of the two advances. Nonetheless, coordinating SDN and NFV is trying because of the assortment of entwined network components included and the intricate association among them. In this article, we attempt to handle this difficult issue by introducing a building structure called Software-Defined Network Virtualization (SDNV). In view of this system, we likewise examine key specialized difficulties to acknowledging SDN-NFV mix and recognize some significant themes for future examination, with a desire to excite the exploration local area's advantage in emerging area.

C.Software-Defined Network Function Virtualization: A Survey - Diverse proprietary network appliances increase both the capital and operational value of service providers, meantime causing some problems in network ossification. NFV is proposed to value those problems by implementing network functions as clean programming commodity and common hardware. NFV allows adaptable deployment, and centralized management of imaginary network function. Included with SDN, product characterized NFV design additionally provide spry traffic directing and combined improvement of organization capacities and capital. This architecture benefits a vast scope of services (e.g., service chaining) and is changing into dominant form of NFV. At that time, it gives noteworthy perspective on the from middlebox to NFV inclusion finally, we present NFV's crucial challenges and important agreements, as well as its potential exploration directions based on different applications domains.

\section{PROPOSED METHODOLOGY}

Program based network by pure division of governing planes and data (b) sharing structure of network to give multitenant which combines traffic and isolation of address, in huge storage center organizing and (c) replaced methods that natively run on a particular system, with the software-realization that run on server - in recent years, both industry and the researcher have given it a lot of consideration over Software Defined Networks (SDN), Network Virtualization (NV), and Network Function Virtualization (NFV) are used to describe these three concepts. This research explores these three principles in detail, which contains how SDN can support network virtualization. SDN, tied in with implementing concepts of modules to network control, the network designer now has the authority to refactor the control plane. This module has seen use in a variety of fields, including network virtualization. This project begins with a review of software defined networking from various perspectives. The survey of SDN is accompanied by a discussion of how SDN plays a key role in NV and NFV. Finally, this study has a positive impact on the environment. Various personal network raise service providers' earnings and running costs while also causing network hardening issues. To executing network functions as native software on commodity and general hardware, network function virtualization (NFV) is suggested. NFV allows for flexible start-up and centralised management of virtual network functions. The software specified NFV architecture, when combined with SDN, allows for more agile traffic steering and mutual enhancement of network operation and properties. This structure aids a wide range of applications (e.g., Service chaining) and is quickly becoming the most popular type of NFV. The implementation of NFV under the Software-defined NFV architecture is examined in depth in this paper. With an emphasis service as a means of application we begin by introducing software-defined NFV architecture as the state of the art in NFV, as well as the relations between NFV and SDN. We then have a historical perspective on the involvement from middlebox to NFV. Then, we discuss the challenges that NFV faces and related solutions, as well as potential research possibilities in different application areas.

\subsection{Block Diagram}

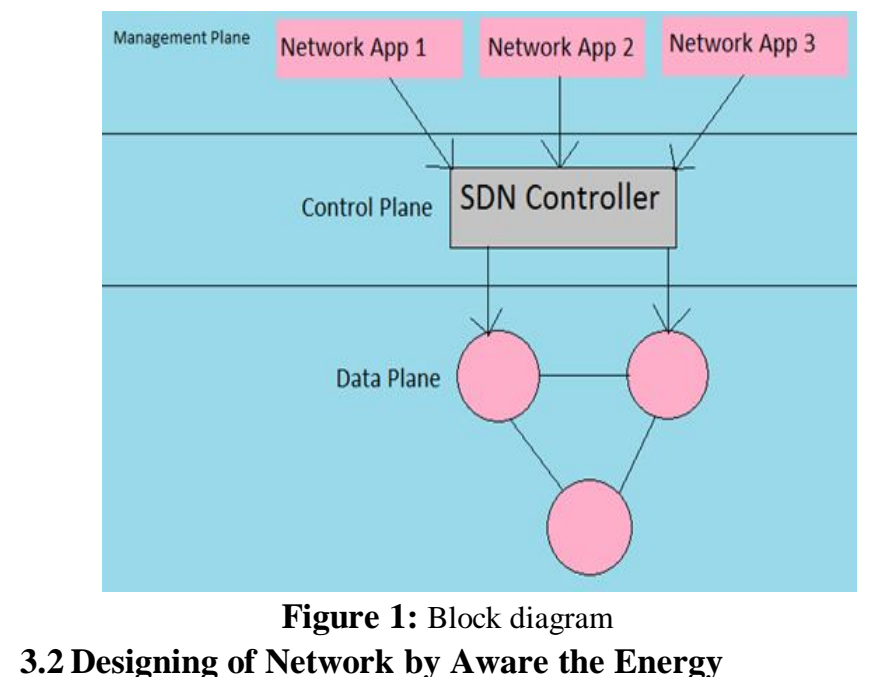

A critical part of the future organization strategy is to build infrastructure that is harmless to the environment network infrastructure by reducing energy use. As shown in figure 1 Network resource virtualization, combined with adaptable SDN control and management, provides enormous potential for energy-efficient networking; however, this advantage has yet to be fully utilized. A test to energy-mindful NFV-SDN mix recline the assortment has interlaced network components which should be considered as the problem, counting the two foundations and administration capacities 
on both information and power/the board planes. For instance, VSF/VN inserting onto arrange and register foundations ought to limit energy utilization while meeting administration quality necessities. Energy-mindful VSF synthesis necessities to accomplish ideal equilibrium among energy utilization, resource use, and service production. Along these lines, try all-encompassing perspective on SDN-NFV joining given by the SDNV structure to encourage energy-mindful which will be future of the network configuration is a fascinating point for future research

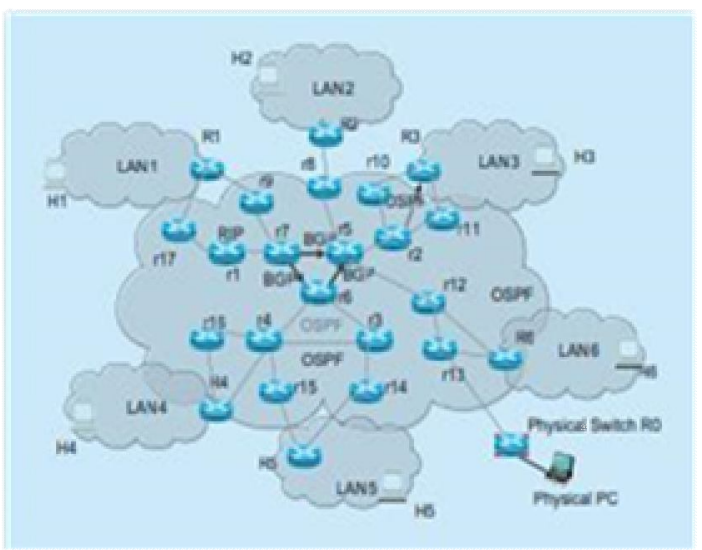

Figure 2: Structure of Connected Networks

Future proof communication networks are relied upon to be executed on virtualized infrastructure as shown in figure 2 where network capacities are conveyed on virtual machines rather than current exclusive hardware. Moving away from an architecture that depends on a huge number of secret elements that are furnished with particular network hardware and pre-stacked with specific software to another architecture comprising of a "white box" running a huge number of particular network programming gives off an impression of being the prevailing decision and the bearing in current and future correspondence and registering frameworks. This is additionally needed to help the $5 \mathrm{G}$ vision, requiring another network architecture that coordinates adaptable, progressively configurable organization components to give on-request modified administrations to traffic requests that might be dynamic in reality, all while supporting heterogeneity and variety. Towards acknowledging a particularly mechanical change in perspective software defined networking (SDN) and network function virtualization (NFV) has been demonstrated as two promising advances for overseeing future organizations. With their colossal advantages, including diminishing operational expenses, better asset utilisation, and simpler administration requirements, the adoption of such innovations is gaining traction. Expanding network asset use and lowering operational costs have traditionally been important goals when forming the board of directors and control. By relocating network capacities from dedicated equipment to virtual machines operating on ware equipment, NFV finds even more adaptability. As a number of business and innovation factors converge, SDN has emerged as a critical catalyst for growth and improvement in systems administration as a few market and innovation factors converge. Among these are the development of cloud apps and services through large enterprises and cloud providers, as well as an emphasis on connected networks. (process/storage/network), and software-defined data-center.

\section{RESULT DISCUSSION}

The intricacy and mix of software and hardware in data-centers raise the trouble to screen and troubleshoot execution issues in a virtualized environment. A PC network is a mind-boggling system, and a disappointment of a single element can affect the system all in all network clients experience: from wrong network arrangements or virtual machine settings to basic workers and network gadgets issues. Checking the network well-being can assist with recognizing such issues, thus to investigate network suitably if there should arise an occurrence of disappointments. For the most part, checking can be characterized as an interaction of information assortment and handling. All the more explicitly, checking in the area of system networking infers a few sub processes

\begin{tabular}{|c|c|c|c|c|c|c|c|}
\hline Feature solution & MeSDN & CSDN & IISDN & NetShare & SottRAN & OpenRAN & MCORD \\
\hline \multicolumn{8}{|l|}{$\begin{array}{l}\text { Network as a Ser- } \\
\text { vice (NaSS) }\end{array}$} \\
\hline NFV abstractions & $\sqrt{ }$ & $\sqrt{ }$ & $\sqrt{ }$ & $\sqrt{ }$ & & & $\sqrt{ }$ \\
\hline Heterogeneity & l & & $\sqrt{ }$ & & $\sqrt{ }$ & $\sqrt{ }$ & $\sqrt{ }$ \\
\hline $\begin{array}{l}\text { Service provider } \\
\text { Network }\end{array}$ & & $\sqrt{ }$ & l & $\sqrt{ }$ & & & \\
\hline Scalability & $\sqrt{ }$ & $\sqrt{ }$ & $\sqrt{ }$ & l & l & $\sqrt{ }$ & $\checkmark$ \\
\hline Reliability & & & & r & & & $\sqrt{ }$ \\
\hline Implementation & & & & $\checkmark$ & & & $\checkmark$ \\
\hline Evaluation-Testing & & $\sqrt{ }$ & l & V & $\checkmark$ & l & l \\
\hline TRL & 6 & 4 & 4 & 6 & 6 & 6 & 7 \\
\hline Network part & EPC \& RAN & EPC \& RAN & EPC \& RAN & RAN & RAN & RAN & EPC \& RAN \\
\hline
\end{tabular}

Table 1: Characteristics of Defined Network

The development of the deliberate iPerf throughput over the long run between the VMs for the various settings at $100 \%$ packet sampling rate. As shown in table 1 our outcomes show that port mirroring, where don't leave the kernel space, has the most minimal expense, diminishing the performance of the TCP transfer by $30 \%$. We notice additionally that the correspondences between client space and kernel space vigorously punish the exchange throughput. In fact, in setting 1 , where packets are just sent to the client space, diminishes the TCP throughput by $40 \%$, while furthermore perusing the packet's contents, by up to $60 \%$ (setting 2). Also, all further packets handling executed by sFlow (for example managing, amassing and sending out to the sFlow collector) is generally modest as it just reductions the throughput by $3 \%$. Figure $2 b$ shows the average estimated iPerf throughput at various 
sampling rates. The drop-in execution relies upon the sampling rate and intently matches with the following law:

$$
T^{*}=\min \left(T, \frac{T_{m}(0)}{1+\alpha s}\right) \text {. }
$$

This outcome persuades the analytical model portrayed in the accompanying section, which attempts to catch the region where the performance of the server isn't affected by sampling, for the two cases (I) sFlow in the physical worker, and (ii) sFlow outside the physical server and came to by port mirroring.

$$
T_{m}(s)=\frac{T_{m}(0)}{1+\alpha s}, \quad T^{*}=\min \left(T_{+} \frac{T_{m}(0)}{1+\alpha s}\right) .
$$

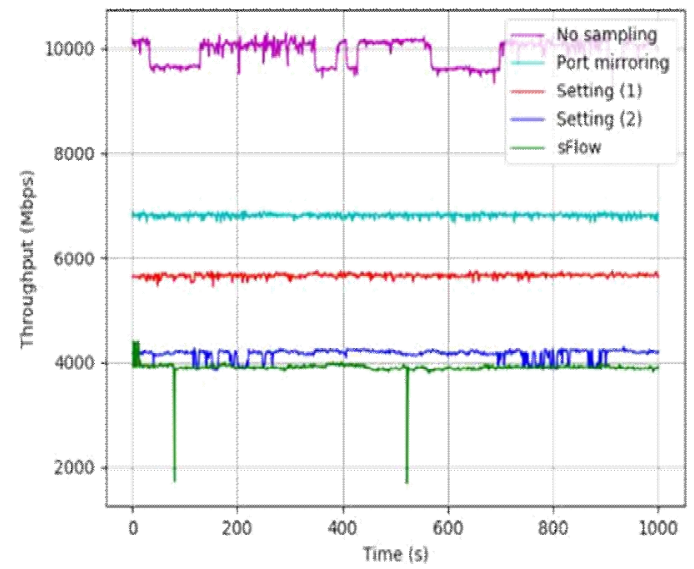

Figure 3: Variation in throughput time at rate of sampling at $100 \%$ for mirroring the port and distinct user program

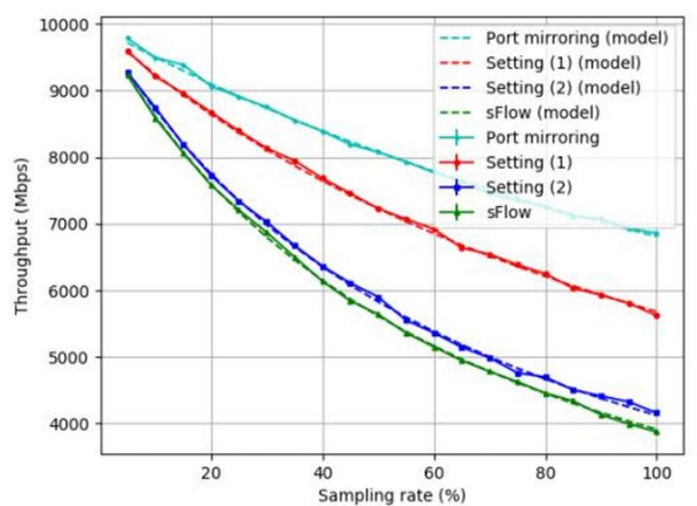

Figure 4: Achievement most of feasible iPerf throughput of vs. rate of sampling for mirroring of port and distinct user

Information Collection: there exist two different ways of information collection: inactive and dynamic. The dynamic measurement reproduces client's behavior by infusing test traffic into the network for observing purposes (e.g., SDN rule checking) as shown in figure 3 while inactive measurement suggests perception of ongoing clients traffic (e.g., Sampling Techniques) as shown in figure 4 and its investigation throughout some timeframe. Information collection doesn't reject a mixture of both. Point by point analysis of these two techniques is introduced. The screenshot of network measurement is shown in figure 6, 7, 8, 9 .

Pre-processing of data: Gathered information is ready for additional stages (organizing, naming, bundling, and so forth)

Transmission of data: arranged information is being send for the analysis as shown in figure 5 .

Examination of analysis: investigation stage, when received information is researched and a wide range of measurements are fabricated.

Data introduction: the results of information analysis are introduced or envisioned for additional insight, giving a view on the network.

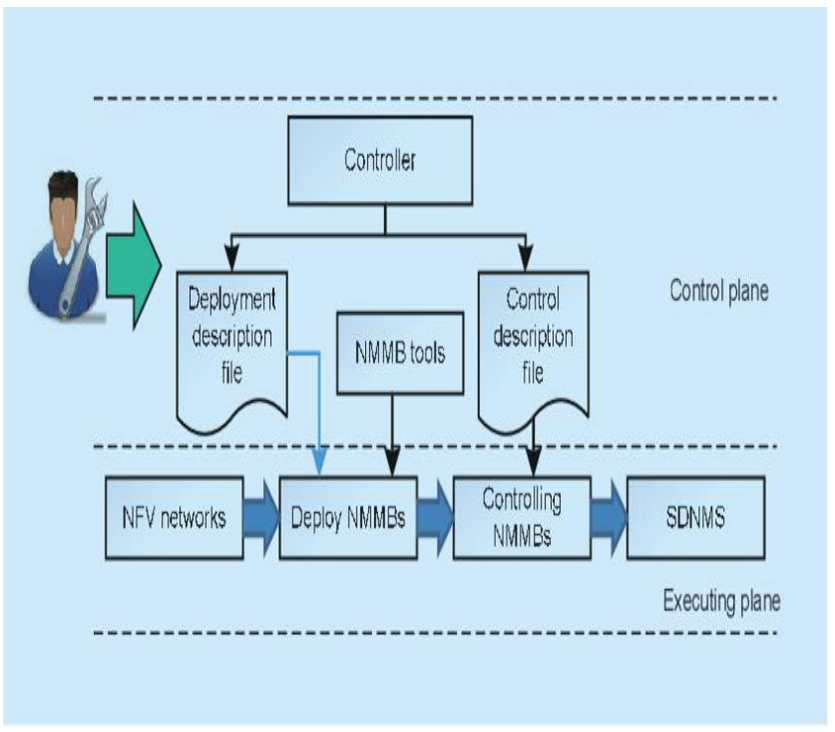

Figure 5: Architecture of Network Middle Box Layer

\subsection{Screen Snippets}

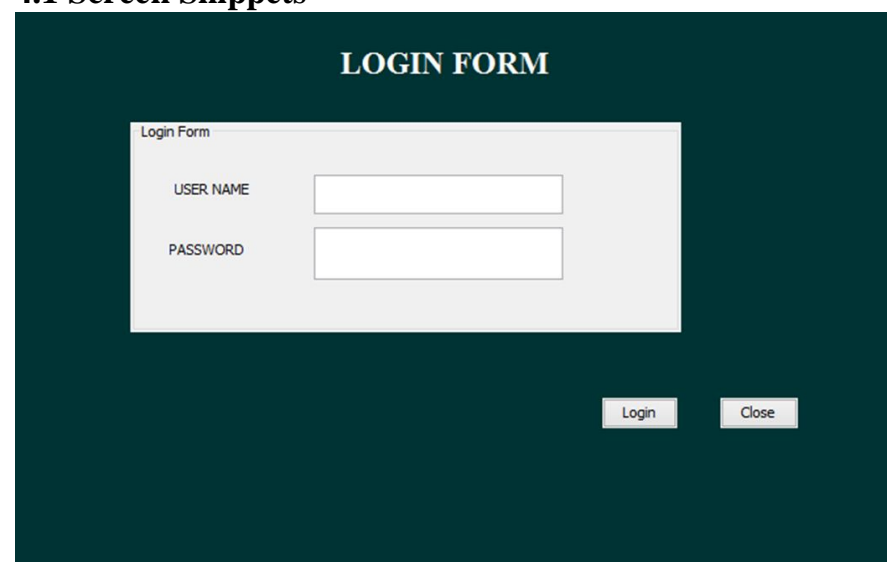

Figure 6: Admin Login 


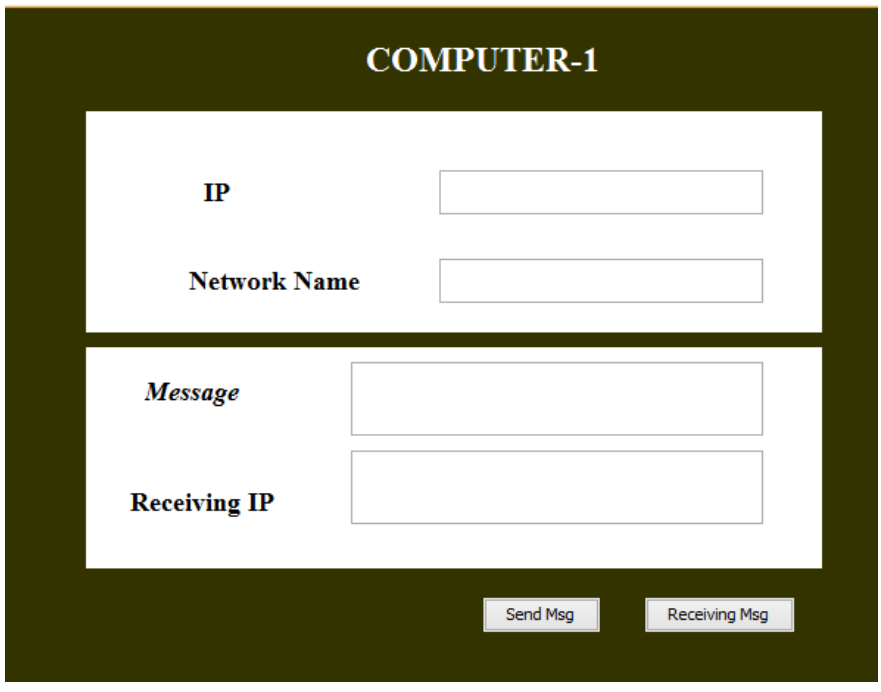

Figure 7: Virtualized Computer Node for sending message

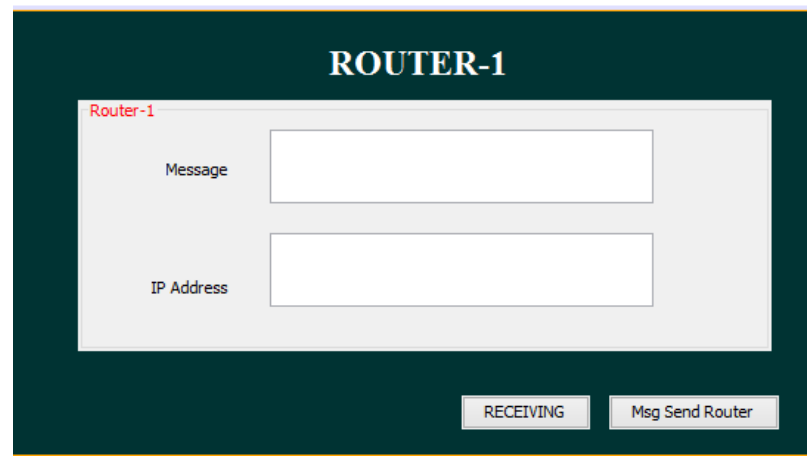

Figure 8: Virtualized Router to verify the sent message passing through SDN Controller or not

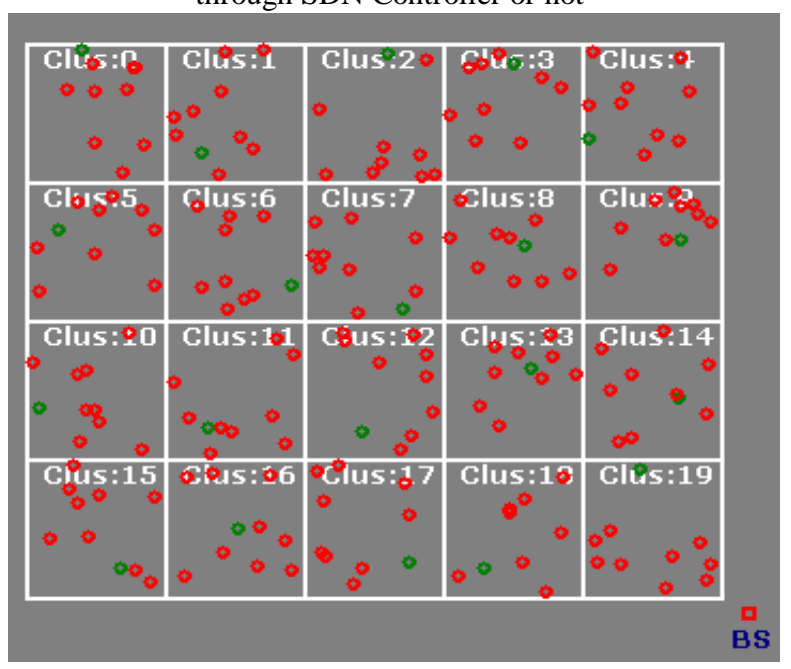

Figure 9: Measurement system deployed in the cluster

\section{CONCLUSION}

The problem was solved by combining SDN and NFV in networks and adding an architecture that combines the core principles of the two ideal models. We first spoke about how SDN and NFV could support, then built a 2-D design to show that how SDN and NFV rely on principles, but we concentrated on measurements separately. Following the
SDNV framework, we also address key technological issues in SDN-NFV collaboration and described quite possible topics for upcoming study. We acknowledge that SDNV structure provides useful supervisions for integrating hard work done for research from various perspectives in order to achieve the shared objective of coordinating SDN and NFV in networks.

\section{REFERENCES}

1. D. Kreutz, F. Ramos, P. Verissimo, C. E. Rothenberg, S. Azodolmolky, and S. Uhlig, Software-Defined Networking: A Comprehensive Survey, Proceedings of the IEEE, vol. 103, no. 1, Jan. 2003, pp. 14-76.

2. NM M. K. Chowdhury and R. Boutaba, A Survey of Network Virtualization, Elsevier Computer Networks Journal, vol. 54, no. 5, Apr. 2010, pp. 862-876.

3. ETSI NFV ISG, Network Function Virtualization Introduction White Paper, Proc. of SDN and OpenFlow World Congress, Oct. 2012.

4. J. Liu, S. Zhang, N. Kato, H. Ujikawa, and K. Suzuki, Device-to-Device Communications for Enhancing Quality of Experience in Software Defined Multi-tier LTE-A Networks, IEEE Network Magazine, vol. 29, no. 4, Jul. 2015, pp. $46-52$.

5. M. Casado, T. Koponen, S. Shenker, and A. Tootoonchian, Fabirc: A Retrospective on Evolving SDN, Proc. of the 1st Workshop on Hop Topics in Software-Defined Networks (HotSDN'12), Aug. 2012.

6. M. Casado, T. Koponen, S. Shenker, and A. Tootoonchian, Software-Defined Internet Architecture: Decoupling Architecture from Infrastructure, Proc. of the 11th ACM Workshop on Hot Topics in Networks (HotNet'12), Oct. 2012.

7. R. Sherwood, G. Gibb, K.-K. Yap, G. Appenzeller, M. Casado, N. McKeown, and G.

Parulkar, FlowVisor: A Network Virtualization Layer, OpenFlow Switch Consortium, Tech.

Rep, 2009.

8. D. Drutskoy, E. Keller, and J. Rexford, Scalable Network Virtualization in Software-Defined Networks, IEEE Internet Computing, vol. 17, no. 2, Mar. 2013, pp. 20-27.

9. T. Wood, K. Ramakrishnan, J. Hwang, G. Liu, and W. Zhang, Toward a Software-Based Network: Integrating Software Defined Networking and Network Function Virtualization, IEEE Network Magazine, vol. 29, no. 3, May 2015, pp. 36-41.

10. W. Ding, W. Qi, J. Wang, and B. Chen, OpenSCaaS: an Open Service Chain as a Service Platform toward the Integration of SDN and NFV, IEEE Network Magazine, vol. 29, no. 3, May 2015, pp. 30-35.

11. J. Matias, J. Garay, N. Toledo, J. Unzilla, and E. Jacob, Toward an SDN-enabled NFV Architecture, IEEE Communications Magazine, vol. 53, no. 4, Apr. 2015, pp. 187-193.

12. Open Network Foundation, ONF Techical Report TR-518: Relationship of SDN and NFV, Oct. 2015. 